\title{
AUTONOMÍA CURRICULAR EN EDUCACIÓN BÁSICA. UNA PROPUESTA DE INNOVACIÓN EN EL MODELO EDUCATIVO 2017 EN MÉXICO
}

\author{
Curriculum Autonomy in Basic Education.
}

A Proposal for Innovation in the Educational Model 2017 in México

Jorge Eduardo Martínez Iñiguez ${ }^{1}$

ORCID: 0000-0002-8833-5600

Sergio Tobón ${ }^{2}$

ORCID: 0000-0001-5543-9131

Odete Serna Huesca ${ }^{3}$

ORCID: 0000-0001-5482-5620

José Antonio Gómez González ${ }^{4}$

ORCID: 0000-0003-0717-7696

${ }^{1}$ Universidad Autónoma de Baja California, Mexicali, Baja California, México.

${ }^{24}$ Centro Universitario CIFE, Cuernavaca, Morelos, México.

${ }^{3}$ Escuela Normal Superior de México, Estado de México, México.

Correos: jorge.martinez43@uabc.edu.mx; stobon5@gmail.com; odetesh@hotmail.com; tony091011@gmail.com

Recibido: 16/11/2019

Aceptado: 07/05/2020

Resumen: El propósito del estudio es analizar el concepto de autonomía curricular en la educación básica mexicana, en el marco del Modelo Educativo 2017 y desde una postura socioformativa. Para ello, se realizó una investigación documental que siguió los ocho ejes de análisis de la cartografía conceptual. Como resultado se destaca que la autonomía curricular desde la socioformación debe promover prácticas educativas que involucren a toda la comunidad educativa para trabajar de manera colaborativa en la resolución de problemas de contexto y en la formación integral del alumno. En conclusión, se sugiere desarrollar nuevos estudios que permitan desarrollar prácticas educativas que aborden la autonomía curricular desde el marco de la responsabilidad, con énfasis en la mejora de las condiciones de vida de los estudiantes y de la sociedad.

Palabras clave: autonomía curricular; Modelo Educativo 2017; educación básica; socioformación. 
Abstract: The purpose of study is to analyze the concept of curriculum autonomy in Mexican basic education within the framework of the Educational Model 2017 and from a socioformative stance. For this, a documentary research was carried out that followed the eight axes of conceptual mapping analysis. As a result, curricular autonomy from socioformation should promote educational practices that involve the entire educational community to work collaboratively in the resolution of context problems and in the integral formation of the student. In conclusion, it is suggested to develop new studies that allow the development of educational practices that address curricular autonomy from the framework of responsibility, with emphasis on improving the living conditions of students and society.

Keywords: curriculum autonomy; Educational Model 2017; basic education; socioformation.

\section{Introducción}

La educación básica, al igual que la media superior, conforman la educación obligatoria en México. Esta tiene como finalidad brindar a los diversos sectores de la sociedad una formación integral con calidad y equidad (Instituto Nacional para la Evaluación de la Educación, 2018; Secretaría de Educación Pública, 2013). El pasado sexenio presidencial México (2012-2018) implementó la reforma educativa 2013, que reformó el artículo 3. ${ }^{\circ}$ de la Constitución Política de los Estados Unidos Mexicanos y realizó modificaciones a la Ley General de Educación, además de crear la Ley del Servicio Profesional Docente y la Ley del Instituto Nacional para la Evaluación de la Educación (Secretaría de Educación Pública, 2013; Instituto Nacional para la Evaluación de la Educación, 2015a, 2016). Esto tuvo la finalidad de fortalecer el sistema de evaluación, así como promover de la mejora continua del sistema educativo mexicano (Instituto Nacional para la Evaluación de la Educación, 2016).

Dentro de la reforma educativa 2013, uno de los cambios más notorios y controversiales en la búsqueda de mejorar la calidad educativa fue lo referente a la instauración del servicio profesional docente. Este, basado en un sistema complejo de evaluación, estableció los mecanismos para el ingreso, la promoción, el reconocimiento y permanencia del docente en el servicio público de la educación obligatoria en México (Barragán y González, 2015; Instituto Nacional para la Evaluación de la Educación, 2015b). Dicha implementación llegó a ser considerada por diversos actores educativos como una reforma de corte laboral sobre la función docente (Barragán y González, 2015), así como la culminación de una serie de reformas educativas neoliberales implementadas en los últimos sexenios presidenciales, que derivaron de la puesta en práctica de recomendaciones emitidas por organismos internacionales como la Organización para 
la Cooperación y el Desarrollo Económicos (OCDE) y el Banco Mundial (BM), entre otros (Coll, 2013; López, 2013; Navarro, 2013).

De acuerdo con el rumbo que fue tomando, pareciera que la reforma educativa 2013 estuvo alejada de un planteamiento pedagógico (Coll, 2013; López, 2013; Navarro, 2013). Empero, en el 2017, la Secretaría de Educación Pública (SEP) presentó tres documentos que vendrían a dar una nueva identidad al currículo de la educación obligatoria acorde con los retos actuales de la sociedad mexicana. Dichos documentos fueron: 1) Los fines de la educación en el siglo XXI; 2) el Modelo Educativo 2017 y 3) la propuesta curricular para la educación obligatoria 2017 (Abellán, 2016).

Antes de concluir la administración federal de Enrique Peña Nieto, se puso en funcionamiento su propuesta pedagógica en el ciclo escolar 2018-2019. Dentro del currículo de la educación básica en México, un componente que se consideró innovador en el marco del Modelo Educativo 2017 fue la llamada autonomía curricular, que consistió en otorgar un mayor grado de flexibilidad a las escuelas del sistema público obligatorio para que estas pudieran tomar sus decisiones y así atender las necesidades e intereses específicos de cada uno de sus alumnos (Diario Oficial de la Federación, 2018).

Con la entrada en funciones del gobierno federal encabezado por Andrés Manuel López Obrador (2018-2024) se generarían cambios sustantivos enfocados a revertir la reforma educativa 2013. El 15 de mayo de 2019 quedaría asentado en el Diario Oficial de la Federación lo siguiente: 1) reforma al artículo 3. ${ }^{\circ}$ de la Constitución Política de los Estados Unidos Mexicanos, con la finalidad de basar la educación en un enfoque de derechos humanos; 2) dejar sin efectos la Ley General del Servicio Profesional Docente que demeritó la labor del profesorado; y 3) abrogar la Ley del Instituto Nacional para la Evaluación de la Educación (Diario Oficial de la Federación, 2019a).

De igual forma, para el ciclo escolar 2019-2020 se establecieron modificaciones al Modelo Educativo 2017 de forma provisional (Secretaría de Educación Pública, 2019), en tanto se construye por completo la denominada Nueva Escuela Mexicana, con un modelo que será aplicado a partir del ciclo escolar 2021-2022. Sin embargo, aunque en la actualidad se deje de hacer énfasis en la autonomía curricular, prevalece en la nueva modificación a la Ley General de Educación la corresponsabilidad social de los actores educativos en el aprendizaje de los estudiantes, en los diferentes niveles de acción — escolares y comunitarios- (Diario Oficial de la Federación, 2019b). 
En este sentido, se vuelve recomendable que las acciones a implantarse dentro de las comunidades escolares estén fundamentadas con enfoques que consideren el contexto y la realidad de las escuelas mexicanas, siendo el enfoque educativo de la socioformación una propuesta que sustente el establecimiento de nuevos proyectos escolares.

La socioformación es una perspectiva educativa que tiene sus raíces latinoamericanas (Tobón, 2002) y se orienta a formar ciudadanos de manera integral, por lo que considera importante abordar los problemas de contexto de manera colaborativa, con emprendimiento, estableciendo un proyecto ético de vida, con metacognición y apoyándose en la utilización de las tecnologías de la información y la comunicación (Tobón, 2017). Esto sin duda hace posible la aplicación de la perspectiva socioformativa dentro de la aplicación y desarrollo de la autonomía curricular propuesta por la SEP para la educación básica en México. Empero, es ineludible estudiar dicho planteamiento educativo si se quiere contar con prácticas educativas exitosas.

Como resultado del cambio de gobierno, y a causa del contexto actual que envuelve al sistema educativo mexicano, el presente estudio ha planteado las siguientes metas: 1) analizar el concepto de autonomía curricular que estableció la SEP en el Modelo Educativo 2017; 2) articular los elementos del enfoque socioformativo con el concepto de autonomía curricular; 3) establecer una propuesta educativa para desarrollar la autonomía curricular desde una perspectiva socioformativa; y 4) orientar la realización de futuras investigaciones en torno a un elemento educativo que es de novedad para las escuelas que conforman la educación básica en el sistema educativo mexicano.

\section{Metodología}

\section{Tipo de estudio}

De acuerdo con las metas establecidas en el estudio, se llevó a cabo una investigación documental, la cual consiste en revisar lo que otros autores han investigado y publicado sobre un tema o problema de interés y así obtener información que permita conocer más sobre el fenómeno que compete estudiar (Del Cid, Méndez y Sandoval, 2007). Como técnica de apoyo se recurrió a la cartografía conceptual, estrategia de investigación que permite estudiar un tema, concepto, teoría, enfoque o metodología a través de ocho ejes de análisis (ver Tabla 1), de modo de categorizar, contrastar la información y orientar la solución de un problema de contexto (Tobón, 
2012). De igual forma, esta metodología es pertinente para explicar y relacionar un concepto de estudio con un determinado enfoque, como es el caso del presente estudio.

Tabla 1

Ejes de análisis de la cartografía conceptual

\begin{tabular}{ll}
\hline Eje de análisis & Preguntas centrales \\
\hline Noción & $\begin{array}{l}\text { ¿Cuál es la etimología, desarrollo histórico y definición del } \\
\text { concepto de autonomía curricular desde la socioformación? }\end{array}$ \\
\hline Categorización & $\begin{array}{l}\text { ¿A qué clase inmediata mayor pertenece la autonomía } \\
\text { curricular? }\end{array}$ \\
\hline Caracterización & $\begin{array}{l}\text { ¿Cuáles son las características centrales de la autonomía } \\
\text { curricular? }\end{array}$ \\
\hline Diferenciación & $\begin{array}{l}\text { ¿De cuáles otros conceptos cercanos y de la misma categoría se } \\
\text { diferencia la autonomía curricular? }\end{array}$ \\
\hline División & ¿En qué subclase o tipos se divide la autonomía curricular? \\
\hline Vinculación & $\begin{array}{l}\text { ¿Cómo se relaciona la autonomía curricular con otros conceptos, } \\
\text { teorías, procesos sociales y referentes que estén por fuera de la } \\
\text { categorización? }\end{array}$ \\
\hline Metodología & $\begin{array}{l}\text { ¿Cuál es el proceso metodológico para aplicar la autonomía } \\
\text { curricular desde la socioformación? }\end{array}$ \\
\hline Ejemplificación & $\begin{array}{l}\text { ¿Cuál podría ser un ejemplo pertinente de desarrollo de la } \\
\text { autonomía curricular desde la socioformación? }\end{array}$ \\
\hline
\end{tabular}

Fuente: Tobón (2012)

\section{Criterios para la búsqueda y selección de los documentos}

Para la realización de la investigación documental, se establecieron los siguientes criterios:

- Búsqueda de documentos en bases de datos como SciELO, Redalyc, WoS, Scopus y Google Académico. Para esto se utilizaron las palabras clave: socioformación, autonomía curricular, modelo educativo en México.

- Selección de documentos que abordaran temas referentes a: la autonomía curricular, el Modelo Educativo 2017 para la educación obligatoria en México, así como la socioformación. Cabe destacar que no se tomaron en cuenta otras perspectivas educativas debido a las metas establecidas en esta investigación.

- Considerar documentos publicados hasta el año 2020 entre artículos académicos, capítulos de libro, libros, documentos de trabajo y manuales. 


\section{Resultados}

\section{Noción del concepto de autonomía curricular desde la socioformación}

De acuerdo con la Real Academia Española (RAE), 'autonomía' proviene del gr.

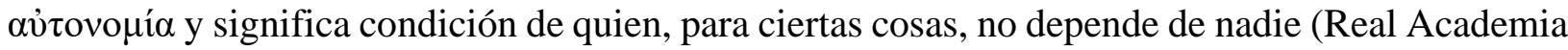
Española, 2014a). Por su parte, 'currículo' es originaria del lat. curricǔlum 'carrera', y es el conjunto de estudios y prácticas destinadas a que el alumno desarrolle plenamente sus posibilidades (Real Academia Española, 2014b). En este sentido, y como consecuencia de la globalización y del modelo económico neoliberal, en las últimas décadas — particularmente, en la década de 1990 - diversos organismos internacionales, con el discurso de buscar elevar la calidad de la educación, recomendaron a las naciones promover, implementar y fortalecer la autonomía de los centros escolares. Esto ha derivado en la gestión de una serie de reformas educativas encaminadas a cumplir con dicho fin, extendiéndose hasta llegar a la región de América Latina (Concha, 2005; Gorostiaga y Tello, 2011).

De acuerdo con Ortega (2017), la autonomía curricular tiene una tradición de décadas de aplicación en países latinoamericanos como El Salvador y Honduras, así como en países del continente europeo (como en España). Sin embargo, si lo que se busca con su implementación es elevar los resultados de los aprendizajes en pruebas estandarizadas como la del Programa Internacional de Evaluación de los Alumnos (PISA), esto no siempre sucede.

En el caso de México, primeramente se iniciaron en 1978 procesos de descentralización educativa, que culminarían en 1992 con la firma del Acuerdo Nacional para la Modernización de la Educación Básica (ANMEB), que delegó a las entidades federativas la responsabilidad de operar administrativamente las escuelas y personal docente y administrativo de la educación básica (preescolar, primaria y secundaria) y normal (Villanueva, 2010). Posteriormente, luego de un diagnóstico situacional, en el 2010 la OCDE realizó 15 recomendaciones con la intención de elevar la calidad de la educación mexicana (Organización para la Cooperación y el Desarrollo Económicos, 2010). Dentro de estas, destaca lo referente a brindar un mayor grado de autonomía escolar a las escuelas mexicanas.

Lo planteado por la OCDE se vería expresado dentro de la reforma educativa 2013, y la autonomía de gestión escolar quedaría asentada en una serie de documentos normativos, tales como la reforma al artículo 3. ${ }^{\circ}$ de la Constitución Política de los Estados Unidos Mexicanos (Diario 
Oficial de la Federación, 2017); el Programa Sectorial de Educación 2013-2018 (Secretaría de Educación Pública, 2013); la modificación a la Ley General de Educación en el 2013 (Diario Oficial de la Federación, 2013); el Acuerdo 716 por el que se establecen los lineamientos para la constitución, organización y funcionamiento de los Consejos Escolares de Participación Social en la Educación (Diario Oficial de la Federación, 2014a) y el acuerdo número 717 por el que se emiten los lineamientos para formular los Programas de Gestión Escolar (Diario Oficial de la Federación, $2014 b)$, entre otros.

Lo antes expuesto plantea todo en referencia a la autonomía de gestión escolar; sin embargo, en cuanto a libertad pedagógica, el Modelo Educativo 2017 (Diario Oficial de la Federación, 2018) aborda de manera explícita el término de autonomía curricular, con lo que se propuso brindar un mayor grado de flexibilidad a la escuelas mexicanas para que tomen sus decisiones tendientes a responder a las necesidades e intereses específicos de sus alumnos, considerando sus recursos y capacidad de gestión escolar.

$\mathrm{Al}$ respecto, es importante mencionar que la SEP en México no establece una definición propia del concepto de autonomía curricular. No obstante, desde un enfoque socioformativo, esta es entendida como el conjunto de prácticas educativas que se implementan de manera colaborativa entre los diversos actores escolares —docentes, autoridades educativas, organizaciones sociales, padres de familia y alumnos - para lograr la formación integral de los estudiantes con base en la construcción de un currículo propio, acorde con las necesidades e intereses de los alumnos, considerando los problemas y retos del contexto en el marco del ejercicio de la responsabilidad y tomando como base un diagnóstico situacional amplio (Tobón, 2017; Vázquez et al., 2017). Esto se propone mediante la metodología de clubes, que son espacios de trabajo colaborativo mediados por un docente, con alumnos de diferentes grados, un plan de acción específico y un determinado horario de trabajo dentro de la jornada escolar (Diario Oficial de la Federación, 2018). Cabe mencionar que estos clubes duran un ciclo escolar y pueden renovarse en el período escolar siguiente.

\section{Categorización del concepto de autonomía curricular}

La autonomía curricular, dentro de la propuesta 2017, es uno de los tres componentes que articula el currículo de la educación básica en México (Secretaría de Educación Pública, 2017b). Es parte esencial del planteamiento curricular establecido por el modelo educativo para la 
educación obligatoria, con la intención de permitir a los centros escolares adaptar los contenidos educativos de acuerdo con el contexto que envuelve a la comunidad escolar y las necesidades específicas del alumno.

Cabe destacar que el Modelo Educativo 2017 es parte de la reforma educativa 2013, la cual elevó a nivel constitucional la responsabilidad del gobierno por mejorar la calidad de la educación, con la intención de que los alumnos respondan a los retos que establece la sociedad en pleno siglo XXI (Secretaría de Educación Pública, 2017a), así como incentivar el desarrollo humano, social, político y económico de México (2017b). Empero, en la práctica, pareciera que las autoridades educativas mexicanas, más que centrarse en lograr una verdadera formación integral del alumno, siguen persiguiendo elevar los índices de resultados satisfactorios en evaluaciones estandarizadas de carácter internacional y nacional, como es el caso de la prueba PISA de la OCDE y del Plan Nacional para la Evaluación de los Aprendizajes (PLANEA) (Secretaría de Educación Pública, 2017a).

Lo anterior, como señalan Chuquilin y Zagaceta (2017), es un condicionante que pudiera dificultar el pleno desarrollo de la autonomía curricular por la inexistencia de un modelo ideal de aplicación para todas las escuelas de México. En ese mismo sentido, Tobón (2017) señala que, si se quiere formar ciudadanos capaces de afrontar los retos y demandas de la sociedad del conocimiento, es necesario dejar de seguir los lineamientos establecidos en diferentes enfoques y modelos educativos que no toman en cuenta las necesidades de contexto propias de la región latinoamericana.

\section{Características que presenta el concepto de autonomía curricular}

De acuerdo con el Modelo Educativo 2017, las características de la autonomía curricular (Secretaría de Educación Pública, 2017a) son las siguientes:

- Educación inclusiva. Busca atender las necesidades e intereses específicos de cada alumno, sin importar factores como religión, lengua materna, condición socioeconómica, entre otros. Al respecto, es de destacar que la educación básica en México casi logra una cobertura universal, empero, aún no se ha logrado obtener una calidad educativa reflejada en los aprendizajes con inclusión y equidad en cuanto a oportunidades y logros educativos (Ramírez, 2015; Secretaría de Educación Pública, 2013). En este sentido, Hernández y Tobón (2016) señalan la importancia de que en las comunidades educativas se promueva 
la participación, atención y éxito de todos los actores educativos, prestando atención especial en aquellos que se encuentren en situación de vulnerabilidad.

- Flexibilidad curricular. Tiende a que las escuelas del país cuenten con mayor autonomía para decidir - a través del Consejo Técnico Escolar y del Consejo Escolar de Participación Social- los contenidos programáticos a desarrollar, con base en las necesidades e intereses manifiestos por los propios alumnos de una comunidad escolar. Esto permite una mayor adaptación de las actividades curriculares, tomando como base las necesidades de contexto expuestas por la comunidad educativa. Desde la socioformación, esto es importante en la búsqueda de la actualización continua del currículo, por lo que se vuelve apremiante que los centros escolares establezcan vínculos de colaboración con diversos sectores de la sociedad, con el fin de enriquecer desde una visión compartida los principales problemas que aquejan a una comunidad educativa, así como las prácticas curriculares a desarrollar dentro de una institución o sistema educativo (Martínez, Tobón y López, 2019).

- Educación de calidad con equidad. Tomando en consideración que el Programa Sectorial de Educación 2013-2018 estableció en su objetivo 1 "asegurar la calidad de los aprendizajes en la educación básica y la formación integral de todos los grupos de la población", la autonomía curricular fue considerada por las autoridades educativas como un componente del Modelo Educativo 2017 que permitiría, gracias a su flexibilidad, fortalecer a la comunidad educativa. Así, estas podrían destinar los recursos necesarios y adaptar el currículo a las necesidades específicas de cada estudiante con el fin de que alcancen su potencialidad en igualdad de oportunidades para todos (Secretaría de Educación Pública, 2017a). Por otra parte, es importante recalcar que una educación de calidad no es aquella que necesariamente debe reflejar buenos resultados en pruebas estandarizas como PISA o PLANEA, como es la tendencia que definió a las autoridades educativas de corte neoliberal en México. Más bien, como lo establece la socioformación, la calidad en educación debe de posibilitar al individuo desarrollar su talento humano y emprender con creatividad las acciones pertinentes que le permitan la resolución de sus problemas de contexto y los de su propia comunidad, así como construir y fortalecer la sociedad del conocimiento, de manera colaborativa y con el apoyo de las tecnologías de la información (Martínez, Tobón, López y Manzanilla, 2020). 


\section{Diferenciación de la autonomía curricular con otros conceptos, enfoques o teorías}

La autonomía curricular propuesta en el Modelo Educativo 2017 se diferencia del currículo tradicional que ha tenido la SEP desde 1921. En sus inicios, una de sus principales prioridades a lo largo del siglo XX fue brindar cobertura educativa y alfabetizar a la población de México desde una perspectiva humanista y sustentada en los ideales de la Revolución Mexicana (Instituto Nacional para la Evaluación de la Educación, 2015b; Secretaría de Educación Pública, 2013, 2017b). Con el paso del tiempo, se ha alcanzado la universalización de la educación primaria, así como la expansión de la matrícula escolar en educación secundaria (Ramírez, 2015). Por ello, es que las autoridades educativas destacaron que en nuestros tiempos hay otras necesidades presentes en la sociedad mexicana, razón por la que a través del Modelo Educativo 2017 se buscó responder a los nuevos retos que trae consigo la denominada sociedad del conocimiento. En este marco, la autonomía curricular es un componente que permite adaptar el currículo a los contextos particulares de cada escuela y alumno en particular (Secretaría de Educación Pública, 2017a).

A diferencia del currículo desarrollado a lo largo del siglo XX, el cual se encontraba estructurado en asignaturas, con rigidez, poca flexibilidad, centrado en la enseñanza de contenidos temáticos estipulados por la SEP y dirigida por un profesor dentro de un aula de clase, con la autonomía curricular se pretende que las comunidades educativas decidan una parte de su currículo. Esto les permite establecer actividades para reforzar los aprendizajes clave, revisar contenidos regionales, realizar actividades de interés personal o desarrollar proyectos que tengan un impacto dentro de su contexto social, promoviendo la formación integral del alumno (Secretaría de Educación Pública, 2017a).

\section{División de la autonomía curricular}

De acuerdo con lo estipulado en el Modelo Educativo 2017, la autonomía curricular se organiza en cinco ámbitos (Diario Oficial de la Federación, 2018; Secretaría de Educación Pública, 2017a), que son:

1) Profundización en la formación académica. Promueve que los alumnos indaguen más a detalle en los temas relativos a las asignaturas de los tres campos formativos de los aprendizajes clave, además de apoyar al alumno que requiera algún tipo de reforzamiento cuando su desempeño académico no sea el adecuado. 
2) Ampliación del desarrollo personal y social. Busca ofrecer oportunidades y espacios de desarrollo acordes a los intereses particulares de cada alumno, con la intención de que estos puedan expresar su creatividad y sus emociones, apreciar el arte o practicar algún deporte.

3) Nuevos contenidos relevantes. Brinda la oportunidad de ofrecer al alumno otros temas que son relevantes en la vida diaria y no se encuentran estipulados en el currículo formal de la educación obligatoria por requerir de profesores especializados para su impartición. Dichas temáticas son: emprendimiento, robótica, finanzas, programación, entre otras.

4) Contenidos regionales y locales. Permite al alumno ampliar sus conocimientos y desarrollar proyectos en temáticas relacionadas a la cultura y tradiciones de la localidad, como artesanías locales, lenguas originarias, autores de la localidad, flora y fauna de la localidad, educación ambiental, entre otras.

5) Impulso a proyectos de impacto social. Busca que la escuela fortalezca sus lazos de colaboración con la comunidad, para que juntos contribuyan a formar de manera integral al alumno por medio del desarrollo de proyectos con impacto social, como pueden ser: reciclaje, impulso de la democracia escolar, cuidado de la salud y del ambiente, cultivo de alimentos, entre otros.

Cabe destacar que cada escuela puede agrupar a sus estudiantes en un espacio curricular dentro de los cinco ámbitos establecidos para llevar a cabo el funcionamiento de la autonomía curricular en la educación básica, pero esto deberá contemplar los intereses y necesidades manifiestas por los alumnos, así como un ejercicio participativo de toda la comunidad escolar a través del Consejo Técnico Escolar y el Consejo Escolar de Participación Social (Secretaría de Educación Pública, 2017a, 2017b). Empero, como señalan Valdés, Carlos y Arreola (2013), las escuelas mexicanas carecen en gran medida de la participación de diversos organismos sociales en las actividades que se desarrollan en el núcleo de las comunidades educativas. Por ello, las autoridades educativas mexicanas deben de establecer mecanismos más eficaces que reactiven la participación social en educación.

Si en las escuelas de la educación básica en México se activa el trabajo colaborativo de diversos actores educativos, se podrá trabajar de manera más eficaz en la identificación de los recursos institucionales disponibles y determinar el grado de madurez organizacional de la institución educativa. Esto serviría como argumento para conformar los clubes pertenecientes a los ámbitos de la autonomía curricular más acordes a las necesidades específicas de una determinada comunidad educativa (Diario Oficial de la Federación, 2018). 
Por ello, es de suma importancia que en las escuelas mexicanas se cuente con el liderazgo de un director que forme a su comunidad para la sociedad del conocimiento (Hernández, Tobón y Vázquez, 2015; Herrera y Tobón, 2017). Ello implica involucrar a todos los actores educativos como autoridades educativas, profesores, docentes, padres de familia, organizacionales sociales y a los propios alumnos - en la toma de decisiones que conlleven a la resolución de sus problemas de contexto (Martínez, Tobón y López, 2019), como puede ser el gestionar la utilización de otras instalaciones fuera del centro escolar o contratar personal especializado para desarrollar las actividades correspondientes a alguno de los ámbitos de la autonomía curricular (Secretaría de Educación Pública, 2017a, 2017b).

\section{Vinculación de la autonomía curricular con otros conceptos, enfoques o teorías}

De acuerdo con el Modelo Educativo 2017 — y con el propósito de articular la educación básica, superar la saturación de contenidos temáticos, promover la transversalidad y el trabajo interdisciplinario, así como el aprender a aprender a lo largo de la vida - la autonomía curricular se vincula con otros dos componentes (Secretaría de Educación Pública, 2017a), que son:

- Campos formativos. Es cada una de las tres cohortes de agrupación de los aprendizajes clave: 1) lenguaje y comunicación; 2) pensamiento matemático; y 3) exploración y comprensión del mundo natural y social. De estos se desglosan las asignaturas que conforman los tres niveles de la educación básica (preescolar, primaria y secundaria) y que proporcionan los contenidos necesarios para la lograr el perfil de egreso deseado, así como servir de referente para las evaluaciones estandarizadas, tanto nacionales como internacionales.

- Desarrollo personal y social. En este componente se promueven conocimientos, habilidades, actitudes y valores en el estudiante de la educación básica, con el fin de que pueda resolver diversos retos. Para ello, se lleva a cabo el trabajo artístico, creativo, corporal y con especial énfasis en actividades que desarrollen habilidades socioemocionales.

\section{Metodología de aplicación de la autonomía curricular desde la socioformación}

A continuación, se presenta un proceso metodológico para desarrollar la autonomía curricular en educación básica desde un enfoque socioformativo: 
1) Establecer el trabajo colaborativo. Si no se cuenta en una escuela, se procede a integrar un Consejo Escolar de Participación Social y un Consejo Técnico Escolar con la intención de asignar roles, establecer acuerdos e involucrar a diversos actores como organizaciones sociales, autoridades educativas, directivos, docentes, estudiantes, padres de familia.

2) Diagnosticar los problemas de contexto. En forma colaborativa, el Consejo Escolar de Participación Social, el Consejo Técnico Escolar y los estudiantes de las escuelas identifican las fortalezas y oportunidades de mejora de la institución educativa, así como de la comunidad que los rodea, con la finalidad de identificar su grado de madurez organizacional y los retos que presentan en su contexto inmediato.

3) Conformar espacios curriculares. Identificadas las problemáticas de contexto y el grado de madurez organizacional en la escuela, se procede de manera colaborativa por todos los miembros de la comunidad educativa a conformar clubes para el desarrollo de la autonomía curricular, con la intención de integrar grupos de estudiantes de acuerdo con sus intereses particulares y sin importar edad ni nivel educativo.

4) Establecer y desarrollar proyectos formativos. De acuerdo con los clubes conformados, cada uno construye su visión compartida con el apoyo de los diversos actores de la comunidad educativa a través del establecimiento de metas a lograr, la gestión de los recursos necesarios y la planeación e implementación de los proyectos formativos a desarrollar paso a paso.

5) Evaluar y promover la mejora continua. Se establecen sesiones de seguimiento de los proyectos formativos en cada club para el desarrollo de la autonomía curricular, con la intención de evaluar la contribución que se tiene a la solución de la problemática de contexto, analizar las principales dificultades a las que se enfrentan los estudiantes, sugerir acciones de mejora a través de asesorías impartidas por parte de supervisores, directivos, docentes o cualquier otro actor educativo especializado en la materia del proyecto realizado.

\section{Ejemplo de aplicación de la autonomía curricular desde la socioformación}

De manera sintética, en la Tabla 2 se muestra un ejemplo que permite visualizar la metodología descrita. 


\section{Tabla 2}

\section{Proceso metodológico para desarrollar la autonomía curricular en la educación básica desde}

\section{un enfoque socioformativo}

\section{País: México.}

Estado: Morelos.

Institución educativa: El Colegio de Cuernavaca.

Ubicación: zona urbana.

Servicio: Los seis grados educativos que conforman la educación primaria.

Número de estudiantes: 130.

Número de docentes: 6 .

Contexto: Institución educativa que presenta múltiples problemas, siendo uno de ellos lo referente al consumo de alimentos con bajo valor nutricional.

\begin{tabular}{cc}
\hline Eje & Descripción
\end{tabular}

1. Trabajo colaborativo
Desde la socioformación se propone realizar el desarrollo de la autonomía curricular en el marco del trabajo colaborativo con los distintos actores educativos y padres de familia, con el fin de tener apoyo mutuo y lograr mayor impacto en los estudiantes. La colaboración implica, esencialmente, conformar un equipo de trabajo, establecer roles, acordar un plan de acción, cumplir con las responsabilidades individuales y aportar al logro de las metas. Por lo anterior, se integraron grupos en los que estaban representados los diversos actores para observar el entorno y establecer roles de trabajo, así como reuniones de seguimiento para todo el ciclo escolar.

2. Diagnóstico Con base en el estudio del contexto externo e interno de la escuela, así como de los diagnósticos de los de los problemas del contexto diversos grupos escolares y las metas curriculares en la formación, se analizaron en colegiado los distintos problemas del contexto escolar. Cada necesidad identificada fue clasificada en el componente de autonomía curricular correspondiente, empero, se encontró que un problema significativo para la comunidad educativa es lo referente al alto consumo de alimentos con bajo nivel nutricional.

3. Conformar espacios curriculares

Después de un análisis pertinente, se determinó que uno de los clubes debía abordar el componente curricular "Impulso a proyectos de impacto social", por lo que se planteó lo siguiente:

Necesidad: se observa en la comunidad estudiantil y en los hogares de estos, un consumo desmedido de alimentos procesados y bebidas azucaradas que no contribuyen a la formación integral de los estudiantes.

Meta: mejorar la alimentación en el hogar y la escuela teniendo como referencia las necesidades calóricas, el plato del buen comer, la jarra del buen beber, los alimentos disponibles en el entorno y los recursos económicos de la familia para conseguirlos, a partir de un análisis del presupuesto mensual.

Lo anterior, permitió establecer los roles de participación en el trabajo colaborativo y las áreas disciplinares que intervendrán, se determinó el conjunto articulado de actividades de aprendizaje, enseñanza, seguimiento y evaluación a realizar por los estudiantes, docentes, directivos y padres de familia. Por ello:

-Los directivos asumieron la encomienda de supervisar el tipo de alimentos que consumían los estudiantes.

-Los profesores integraron una actividad conjunta para trabajar sobre el tema de la alimentación en las asignaturas de Biología, Historia, Geografía y Educación Física, con el propósito de que los alumnos valoraran la riqueza de los alimentos, particularmente los de su comunidad.

-El responsable del centro de cómputo ofreció asesoría a los alumnos en la búsqueda de información.

-Los padres de familia se agruparon para intercambiar ideas sobre su responsabilidad en la alimentación de los adolescentes.

-El médico escolar valoró algunos casos de alumnos que presentaban sobrepeso o peso muy bajo, previa autorización de los padres de familia. 
Continúa Tabla 2. Proceso metodológico para desarrollar la autonomía curricular en la educación básica desde un enfoque socioformativo

4. Establecer y Se formó a la comunidad educativa para que puedan aplicar la metodología de los proyectos desarrollar proyectos formativos formativos. Posteriormente, la dirección escolar establece su proyecto de mejora escolar a través de esta metodología, así como también los profesores de la escuela hacen lo mismo para abordar los aprendizajes claves establecidos en el ámbito de la autonomía curricular.

Se da prioridad a los proyectos formativos de los estudiantes, y así resolver los problemas de contexto relacionados con: las consecuencias de la mala alimentación, el consumo de productos procesados y bebidas azucaradas, la promoción de una sana alimentación, activación física, entre otros. Dichos proyectos, se van desarrollando durante todo el ciclo escolar con la asesoría de los profesores involucrados en el abordaje del componente curricular de "Impulso a proyectos de impacto social".

5. Evaluar y promover la mejora continua
Conforme va pasando el ciclo escolar, se comparten las evidencias de los proyectos con la comunidad educativa, quienes van proponiendo acciones de mejora a cada proyecto desarrollado por los estudiantes.

Para concluir el ciclo escolar, la dirección escolar y los profesores presentan a la comunidad educativa los resultados obtenidos de los proyectos formativos desarrollados por parte de los estudiantes, mismos que reflejaron: 1) reducción de consumo de alimentos procesados en un 25\%; 2) en un $20 \%$, se dejó de consumir bebidas azucaradas; 3 ) se empezó a incluir frutas y verduras en la alimentación personal en un 30\%; 4) se comenzó a realizar actividad física en 4 días de la semana, con una duración de media hora por día; y 5) en las familias de la comunidad educativa, se aumentó el consumo de frutas y verduras en un $25 \%$.

Para finalizar, los estudiantes en compañía de sus profesores y de la comunidad educativa expresan que consideran qué hicieron bien en su proyecto y qué deben hacer para seguir mejorando sus hábitos alimenticios y los de su comunidad.

Fuente: Elaboración propia (2019)

\section{Conclusiones}

Acorde con el estudio realizado, se llega a la conclusión de que la autonomía curricular en la educación básica en México, y dentro del marco del Modelo Educativo 2017, se refiere a la gestión que debe hacer cada escuela para construir e implementar un componente curricular acorde con sus características e intereses de los estudiantes. Se consideran cinco ámbitos de autonomía curricular, para cuya elección se necesita del trabajo colaborativo de diversos actores educativos en la elaboración de un diagnóstico institucional y en la identificación del grado de madurez escolar (Diario Oficial de la Federación, 2018; Secretaría de Educación Pública, 2017a).

Desde el enfoque de la socioformación, se propone focalizar la autonomía curricular a través del trabajo con proyectos en cada uno de los clubes, y así potencializar el trabajo colaborativo de las autoridades educativas, directivos, docentes, padres de familia, estudiantes y diversos organismos de la sociedad. Se busca que se consideren los intereses de todos los 
miembros que conforman e interactúan en una institución educativa, pero también es menester establecer cuál es la contribución a la mejora de las condiciones de vida de la comunidad educativa.

Sin duda, es necesario involucrar a toda la comunidad educativa para que trabaje de manera colaborativa en el diagnóstico de los intereses de los alumnos, como también en la conformación y el funcionamiento de los clubes pertenecientes a los ámbitos de la autonomía curricular (Diario Oficial de la Federación, 2018; Secretaría de Educación Pública, 2017a). El objetivo es que se estén reinventando de manera continua y que, en ellos, los alumnos se formen con motivación y bajo los principios de calidad y equidad propuestos en el discurso del Modelo Educativo 2017 (Secretaría de Educación Pública, 2017a), así como en la búsqueda de una educación humanista y centrada en las necesidades de las personas, tal como se viene construyendo en la denominada Nueva Escuela Mexicana (Diario Oficial de la Federación, 2019; Secretaría de Educación Pública, 2019).

Por último, al ser la autonomía curricular un componente de reciente incorporación y de innovación dentro del currículo de la educación básica en México, es imprescindible el seguir realizando diversas investigaciones que permitan evaluar su operación, desarrollo y grado de pertinencia dentro de las escuelas mexicanas. De este modo, se podrán implementar las acciones de mejora que se consideren en el momento oportuno, y así asegurar que dicho componente les otorgue identidad a las comunidades educativas, además de permitirles resolver sus problemas de contexto más apremiantes.

\section{Referencias}

Abellán, J. (2016). El modelo educativo 2016, entre la simulación y el falso consenso . Revista Latinoamericana de Estudios Educativos, 56(3), 45-84. Recuperado de http://www.redalyc.org/pdf/270/27047597003.pdf

Barragán, A. S., y González, J. H. (2015). Servicio Profesional Docente: una construcción de mercado laboral. En M. A. Navarro, y Z. Navarrete, Educación Comparara Internacional y Nacional (pp. 275-285). México, D.F., México: Sociedad Mexicana de Educación Comparada/Plaza y Valdes Editores.

Chuquilin, J., y Zagaceta, M. (2017). El currículo en la educación básica en tiempos de transformaciones. Revista Mexicana de Investigación Educativa, 22(72), 109-134.

Coll, T. (2013). La reforma educativa, el poder del Estado y la evaluación. El Cotidiano, 179, 43-54.

Concha, C. (2005). Gestión de las Reformas Educacionales en América Latina en los 90. Primeras aproximaciones a un proceso complejo. Revista Electrónica Iberoamericana sobre Calidad, Eficacia y Cambio en Educación, 3(2), 131-153. Recuperado de http://www.rinace.net/arts/vol3num2/art8.pdf 
Del Cid, A., Méndez, R., y Sandoval, F. (2007). Investigación. Fundamentos y Metodología. México D. F., México: Pearson Educación.

Diario Oficial de la Federación. (2013). Decreto por el que se reforman, adicionan y derogan diversas disposiciones de la Ley General de Educación. Recuperado de http://dof.gob.mx/nota_detalle.php?codigo=5313841\&fecha=11/09/2013

Diario Oficial de la Federación. (2014a). Acuerdo número 716 por el que se establecen los lineamientos para la constitución, organización y funcionamiento de los Consejos de Participación Social en la Educación. Recuperado de http://www.dof.gob.mx/nota_detalle.php?codigo=5335232\&fecha=07/03/2014

Diario Oficial de la Federación. (2014b). Acuerdo número 717 por el que se emiten los lineamientos para formular los Programas de Gestión Escolar. Recuperado de http://www.dof.gob.mx/nota_detalle.php?codigo=5335233\&fecha=07/03/2014

Diario Oficial de la Federación. (2017). Constitución Política de los Estados Unidos Mexicanos. Recuperado de http://www.diputados.gob.mx/LeyesBiblio/pdf/1_150917.pdf

Diario Oficial de la Federación. (2018). Acuerdo número 11/05/18 por el que se emiten los Lineamientos para el desarrollo y el ejercicio de la autonomía curricular en las escuelas de educación básica del Sistema Educativo Nacional. Recuperado de http://dof.gob.mx/nota_detalle.php?codigo=5525413\&fecha=07/06/2018

Diario Oficial de la Federación. (2019a). Decreto por el que se reforman, adicional y derogan diversas disposiciones de los artículos $3^{\circ}$., 31 y 73 de la Constitución Política de los Estados Unidos Mexicanos, en materia educativa. Recuperado de https://www.dof.gob.mx/nota_detalle.php?codigo=5560457\&fecha=15/05/2019

Diario Oficial de la Federación. (2019b). Decreto por el que se expide la Ley General de Educación y se abroga la Ley General de la Infraestructura Física Educativa. Recuperado de https://www.dof.gob.mx/nota_detalle.php?codigo=5573858\&fecha=30/09/2019

Gorostiaga, J., y Tello, C. (2011). Globalización y reforma educativa en América Latina: un análisis inter-textual. Revista Brasileira de Educação, 16(42), 363-388. Recuperado de http://www.scielo.br/pdf/rbedu/v16n47/v16n47a06.pdf

Hernández, J. S., Tobón, S., y Vázquez, J. M. (2015). Estudio del liderazgo socioformativo mediante la cartografía conceptual. Revista Iberoamericana de Evaluación Educativa, 8(2), 105-128.

Hernández, H., y Tobón, S. (2016). Análisis documental del proceso de inclusión en la educación. Ra Ximhai, 12(6), 399-420.

Herrera, S. R., y Tobón, S. (2017). El director escolar desde el enfoque socioformativo. Estudio documental mediante la cartografía conceptual. Revista de Pedagogía, 38(102), 164-194.

Instituto Nacional para la Evaluación de la Educación. (2015a). Reforma educativa. Marco normativo. México: LXII Legislatura de la Cámara de Diputados/ Instituto Nacional para la Evaluación de la Educación.

Instituto Nacional para la Evaluación de la Educación. (2015b). Los docentes en México. Informe 2015. México, D.F.: Instituto Nacional para la Evaluación de la Educación.

Instituto Nacional para la Evaluación de la Educación. (2016). Política Nacional de Evaluación de la Educación. México, D.F.: Instituto Nacional para la Evaluación de la Educación. 
Instituto Nacional para la Evaluación de la Educación. (2018). La educación obligatoria en México. Informe 2018. Ciudad de México: Instituto Nacional para la Evaluación de la Educación.

López, M. (2013). Una reforma "educativa" contra los maestros y el derecho a la educación. El Cotidiano, 129, 5576.

Martínez, J. E., Tobón y López, E. (2019). Currículo: un análisis desde un enfoque socioformativo. IE Revista de Investigación Educativa de la REDIECH, 10(18), 43-63. https://doi.org/10.33010/ie_rie_rediech.v10i18.200

Martínez, J. E., Tobón, S., López, E. y Manzanilla, H. M. (2020). Calidad educativa: un estudio documental desde una perspectiva socioformativa. Revista Latinoamericana de Estudios Educativos, 16(1), 233-258.

Navarro, C. (2013). La reforma educativa: despojo y castigo constitucional al magisterio. El Cotidiano, 179, 77-88.

Organización para la Cooperación y el Desarrollo Económicos. (2010). Acuerdo de Cooperación México-OCDE para mejorar la calidad de la educación de las escuelas mexicanas. Recuperado de https://www.oecd.org/edu/school/46216786.pdf

Ortega, F. (2017). Principios e implicaciones del Nuevo Modelo Educativo. Revista Latinoamericana de Estudios Educativos, 47(1), 43-62. Recuperado de http://www.redalyc.org/articulo.oa?id=27050422003

Ramírez, R. (2015). El cambio educativo necesario. Los desafíos de la política. En R. Ramírez, La política educativa del sexenio 2013-2018. Alcances y límites (pp. 9-16). Ciudad de México: Instituto de Investigaciones para el Desarrollo de la Educación/Universidad Iberoamericana.

Real Academia Española. (2014a). Autonomía. En Dicccionario de la Lengua Española. Madrid, España: Real Academia Española.

Real Academia Española. (2014b). Currículo. En Dicccionario de la Lengua Española. Madrid, España: Real Academia Española.

Secretaría de Educación Pública. (2013). Programa Sectorial de Educación 2013-2018. México, D.F., México: Secretaría de Educación Pública.

Secretaría de Educación Pública. (2017a). Nuevo Modelo Educativo para la educación obligatoria. México D. F., México: Secretaría de Educación Pública.

Secretaría de Educación Pública. (2017b). Plan y programas de estudio para la educación básica. Ciudad de México, México: Secretaría de Educación Pública.

Secretaría de Educación Pública. (2019). Hacia una Nueva Escuela Mexicana: Taller de Capacitación. Recuperado de https://educacionbasica.sep.gob.mx/multimedia/RSC/BASICA/Documento/201908/201908-RSCm93QNnsBgD-NEM020819.pdf

Tobón, S. (2002). Modelo pedagógico basado en competencias. Medellín, Colombia: Funorie.

Tobón, S. (2012). Cartografía conceptual: estrategia para la formación y evaluación de conceptos y teorías. México D. F., México: CIFE.

Tobón, S. (2017). Conceptual analysis of the socioformation according to the knowledge society. Knowledge Society and Quality of Life, l(1), 9-35. Recuperado de https://cife.edu.mx/ksql/index.php/ksql/issue/view/1

Valdés, A., Carlos, E. y Arreola, C. G. (2013). Desarrollo de un instrumento para medir la participación de los padres en la educación escolar de los hijos. Revista de Evaluación Educativa, 2(1), 1-18. Recuperado de https://www.researchgate.net/profile/Angel_Valdes3/publication/285871818_Desarrollo_de_un_instrument 
o_para_medir_la_participacion_de_los_padres_en_la_educacion_de_los_hijos/links/5664053e08ae4931cd 605a38.pdf

Vázquez, J. M., Hernández, J. S., Vázquez, J., Juárez, L. G., y Guzmán, C. E. (2017). El trabajo colaborativo y la socioformación: un camino hacia el conocimiento complejo. Revista Educación y Humanismo, 19(33), 334356. Recuperado de http://revistas.unisimon.edu.co/index.php/educacion/article/view/2648/2678

Villanueva, P. (2010). Impacto de la descentralización de la educación básica y normal en México sobre el gasto en nómina magisterial de los estados, 1999-2004. Economía Mexicana. Nueva Época, 19(2), 231-270.

\title{
Contribución autoral
}

a) Concepción y diseño del trabajo; b) Adquisición de datos; c) Análisis e interpretación de datos; d) Redacción del manuscrito; e) revisión crítica del manuscrito.

J. E. M. I. ha contribuido en a, b, c, d.

S. T. ha contribuido en d, e.

O. S. H. ha contribuido en b.

J. A. G. G. ha contribuido en b.

\section{Editora científica responsable}

\author{
Mag. Florencia Deleon
}

